\title{
Tocilizumab, a Humanized Anti-interleukin-6 Receptor Antibody, Ameliorates Joint Swelling in Established Monkey Collagen-Induced Arthritis
}

\author{
Yasushi Uchiyama, Keigo Yorozu, Misato Hashizume, Yoichiro Moriya, and Masahiko Minara* \\ Product Research Department, Chugai Pharmaceutical Co., Ltd.; 1-135, Komakado, Gotemba, Shizuoka 412-8513, Japan. \\ Received January 22, 2008; accepted February 29, 2008; published online March 4, 2008
}

\begin{abstract}
We examined if tocilizumab, humanized anti-interleukin-6 receptor (IL-6R) antibody, can ameliorate joint swelling after the onset of arthritis in collagen-induced arthritis (CIA). CIA was induced by the immunization of bovine type II collagen in female cynomolgus monkeys. Tocilizumab $(30 \mathrm{mg} / \mathrm{kg})$ was administered weekly for 4 weeks after the onset of arthritis. Swelling of 16 proximal interphalangeal (PIP) joints of hands and feet was monitored by measuring the longitudinal and transverse axes of PIP joints and the oval area of each PIP was calculated. Serum was collected once a week after tocilizumab injection and blood chemistry, IL-6, soluble IL-6R (sIL-6R), and anti-tocilizumab antibody were measured. At the end of study, histopathological examination of joints was performed. Tocilizumab clearly reduced joint swelling in all animals without anti-tocilizumab antibody. Histopathological study showed significant decrease in the infiltration of neutrophils into inflamed joints was observed in tocilizumab-treated animals. In conclusion, tocilizumab improved established arthritis in monkey and monkey CIA model is useful for the analysis of anti-arthritic effect of tocilizumab.
\end{abstract}

Key words tocilizumab; therapeutic effect; monkey collagen-induced arthritis

Tocilizumab is a humanized anti-interleukin-6 receptor (IL-6R) antibody. It inhibits the binding of IL-6 to IL-6R and specifically neutralizes IL-6 actions. ${ }^{1)}$ Clinical trials showed that the treatment with tocilizumab significantly ameliorated the disease activity of rheumatoid arthritis (RA), indicating that IL-6 plays important roles in the pathogenesis of RA. ${ }^{2-4)}$ However, the anti-rheumatic mechanism of tocilizumab in RA is still unclear.

There are many papers showing that the onset of arthritis was restrained by the blockade of IL-6. For instance, we reported that the administration of anti-mouse IL-6R antibody starting from the first collagen immunization (before the onset of arthritis) apparently inhibited the development of arthritis in monkey or mouse collagen-induced arthritis (CIA). ${ }^{5,6)}$ Moreover, IL-6-deficiency could inhibit the appearance of arthritis in many arthritis models. ${ }^{7-11)}$ In these experiments, however, IL-6 was inhibited before the onset of arthritis, and the suppressive mechanism was indicated to be due to the suppression of $\mathrm{T}$ cell activation.

However, as anti-rheumatic agents are used in RA patients with arthritis, it is important to study the influence on established joint swelling. In fact, tocilizumab significantly reduced the number of swollen joints and tender joints rapidly in RA patients. ${ }^{2-4)}$ However, when we injected anti-mouse IL-6R antibody after onset of arthritis in mouse CIA and other mouse arthritis models, it did not reduce established joint swelling (unpublished data). Because of inefficacy of anti-IL-6R antibody in mouse models, it was difficult to analyze how anti-IL-6R antibody improved established arthritis in RA patients.

We have reported that tocilizumab prevented the development of monkey CIA. ${ }^{5)}$ There are differences between rodent and monkey CIA regarding the onset site and the duration time of arthritis. Namely, joint swellings in monkey CIA are preferentially in proximal interphalangeal (PIP) joints of hands and feet, and they last more than one month like a human RA. However, those in rodent CIA are preferentially observed in ankles and wrists, and they are transient.

In the present study, we examined if tocilizumab could reduce joint swelling after the onset of arthritis using monkey CIA model.

\section{MATERIALS AND METHODS}

Reagent Tocilizumab (humanized anti-human IL-6R monoclonal antibody, human IgG1) was expressed in Chinese hamster ovary cells and prepared in our laboratories.

Animals Female cynomolgus monkeys (Macaca Fascicularis) weighing $2.42-3.61 \mathrm{~kg}$, age $3-6$ years old were purchased from the Guangxi Research Center of Primate Laboratory Animals, Guangxi, China. Approximately $108 \mathrm{~g}$ of solid food (Teklad Global Certified 25\% Protein Primate Diet, Harlan Sprague Dawley Inc., Indianapolis, IN, U.S.A.) was provided to each animal daily and water was available ad libitum from an automatic supply.

All procedures involving animals were approved by the animal care and use committee of the laboratory, and were performed in accordance with standards published by the National Research Council (Guide for the Care and Use of Laboratory Animals, NIH OACU), the National Institutes of Health Policy on Human Care and Use of Laboratory Animals. The monkeys were housed in cages (in confirmation with NIH requirements) and maintained on a $12 \mathrm{~h}$ light $/ 12 \mathrm{~h}$ dark cycle.

Induction of Collagen-Induced Arthritis The monkey CIA was induced by modifying the method that we reported previously. ${ }^{5)}$ Briefly, bovine type II collagen $(4 \mathrm{mg} / \mathrm{ml}$, Collagen Research Center, Tokyo, Japan) was emulsified in an equal volume of Freund's complete adjuvant (Becton Dickinson, Grayson, GA, U.S.A.). Prepared emulsion was maintained on ice until use. Each animal was administered intradermally with $2 \mathrm{ml}$ of emulsion dorsally (1st sensitization), with a 2 nd sensitization performed in the same manner, 3 weeks afterwards. 
Measurement of Joint Swelling The longitudinal and transverse axes of PIP joints of the fore and hind limbs (without thumb) were measured with calipers (Vernier Caliper, Mitsutoyo Co., Kanagawa, Japan) and the oval area of each PIP was calculated using the following formula.

oval area $=$ longitudinal axis $\times$ transverse axis $\times 3.14 \times 1 / 4$

Mean of oval area of 16 PIP joints was calculated and adopted as individual data. Percent of oval area and joint swelling was calculated using the following formula.

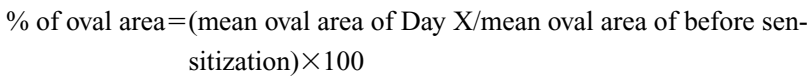

$\%$ of oval area $=($ mean oval area of Day X/mean oval area of before sensitization) $\times 100$

joint swelling $=($ mean oval area of Day $\mathrm{X}-$ mean oval area of before sensitization)/(mean oval area of Day $0-$ mean oval area of before sensitization) $\times 100$

Experimental Protocol At $12 \mathrm{~d}$ after the 2nd sensitization, monkeys were assigned to two groups based on $\%$ of oval area and body weight, to achieve approximately the same degree between the 2 groups. Tocilizumab $(30 \mathrm{mg} / \mathrm{kg}$ ) was injected into the cephalic vein of the forearm of tocilizumab group animals, weekly for 4 weeks (from $3 \mathrm{~d}$ after grouping). For the control group, the same volume of DULBECCO'S PBS (Dainippon Pharmaceutical Co., Ltd., Osaka, Japan) was injected in the same manner. The control and tocilizumab groups consisted of 6 and 8 animals, respectively. Measurement of oval area was undertaken twice prior to dosing (before sensitization and grouping) and 5 times during dosing (Days 0, 7, 14, 21, 28). Bleeding was undertaken once prior to sensitization, and 6 times during dosing (Days 0, 3, 7, 14, 21, 28). Blood was drawn from the femoral vein, with a syringe containing heparin sodium.

Measurement of Anti-tocilizumab Antibody Plasma concentrations of anti-tocilizumab antibody were measured by ELISA, as previously described. ${ }^{5}$ Standard monkey antitocilizumab antibody was purified from sera of tocilizumabtreated cynomolgus monkey.

Blood Chemistry Plasma was obtained by centrifugation immediately after collection. Blood chemical parameters were measured by means of an automatic analyzer JCA-BM8 (JEOL Ltd., Tokyo, Japan). Blood chemical parameters were as follows: C-reactive protein (CRP), aspartate aminotransferase (AST), alanine aminotransferase (ALT), total cholesterol (T-cho), lactate dehydrogenase (LDH), and triglyceride (TG).

Measurement of IL-6 and sIL-6 Receptor Plasma concentrations of IL-6 and soluble IL-6R (sIL-6R) were measured by ELISA kit for human IL-6 and human IL-6R (Biosource Int., Camarillo, CA, U.S.A.), respectively.

Histopathological Examination On day 28, animals were euthanized by exsanguination under anesthesia, by an intravenous injection of sodium pentobarbital solution into the saphenous vein. Both hands and feet were extirpated and fixed in $10 \%$ neutral buffered formalin. After decalcification, slide specimens were prepared by sectioning paraffin-embedded tissue and staining with Hematoxylin-Eosin (HE) stain. Histological grading was carried out using a grading system of $0-3$ ( 0 : no neutrophil infiltration, 1 : very slight, 2 : slight, 3: moderate). Two animals with high concentration of antitocilizumab antibody were excluded.

Statistical Analysis The statistical significance of the differences was analyzed using a software package (Statistical Analysis System: SAS Institute Japan, Tokyo, Japan). $U$ test was used for histopathological examination. The significance level for each analysis was set as $5 \%$.

\section{RESULTS}

Swelling of PIP Joints Joint swelling (\% of oval area) showed wide variation (from 115.88 to $182.14 \%$ in control group and from 112.53 to $195.84 \%$ in tocilizumab group) at the time of first tocilizumab injection (Day 0).

In the control group, joint swelling was almost steady during the administration period in all animals except for one in which joint swelling was increased. On the other hand, in the tocilizumab-treated group, a clear decrease in joint swelling was observed in 6 of 8 animals. This suppressive effect was observed in all severity of joint swelling. In the remaining 2 animals, joint swelling increased transiently on Day 7. However, a decrease in joint swelling was noted in only one animal thereafter (Fig. 1).

CRP Levels All animals exhibited elevated CRP levels on Day 0 (just before tocilizumab injection). In the control group, a time-dependent decrease of CRP was noted in all animals throughout the dosing period. But CRP never disappeared. In contrast, after a single injection of tocilizumab, a remarkable decrease was noted in all animals on Day 3, and the CRP became negative on Day 7. Thereafter, the disappearance of CRP was maintained in all except for 2 animals who produced high concentration of anti-tocilizumab antibody (Fig. 2).

Plasma IL-6 and sIL-6R Levels In all animals, IL-6 was not detectable in plasma prior to sensitization, and elevated production of IL- 6 was observed after onset of arthritis (Day 0). In the control group, IL-6 levels decreased gradually until Day 28, except for one animal which maintained a steady plasma IL-6 level. On the other hand, in the tocilizumab-treated group, plasma IL-6 levels increased re-

Control
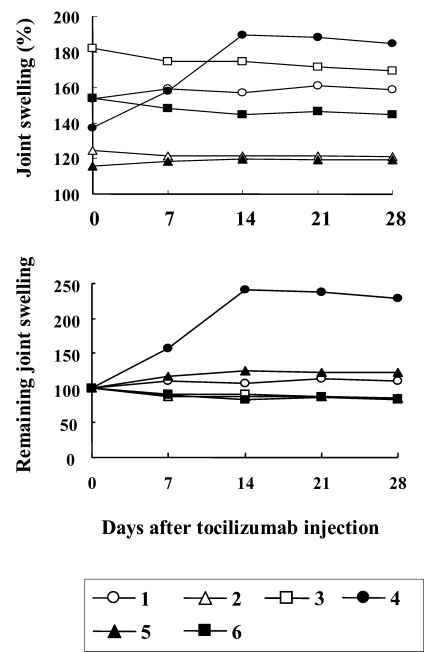

Fig. 1. Swelling of PIP Joints in Tocilizumab-Treated Monkeys

Measurement of joint swelling was carried out 5 times during dosing (Days 0, 7, 14 $21,28)$ according to Materials and Methods. The each symbol indicates individual animal.
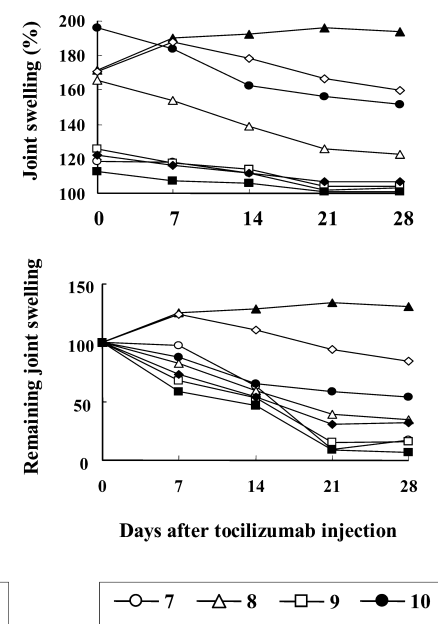

$\neg-11 \rightarrow-12 \multimap-13 \multimap 14$ 
markably until Day 3 or 7 , and thereafter decreased gradually until Day 28 (Fig. 3).

sIL-6R were detectable in plasma prior to sensitization in all monkeys. In the control group, sIL-6R levels remained unchanged from before sensitization through Day 28. On the other hand, in the tocilizumab-treated group, the sIL-6R concentrations increased from Day 3 until Day 28, although in two animals the concentrations decreased from Day 14 or Day 21 (Fig. 3).

Blood Chemistry Regarding AST, ALT, T-cho, LDH, and TG, the change of mean values was in the normal range and there was no animal which had abnormal value throughout experiment (Table 1).

Anti-tocilizumab Antibody Levels Anti-tocilizumab antibody levels were not detected in any animal before dosing of tocilizumab. In only one animal, anti-tocilizumab antibody was not detectable during the dosing period, but anti-
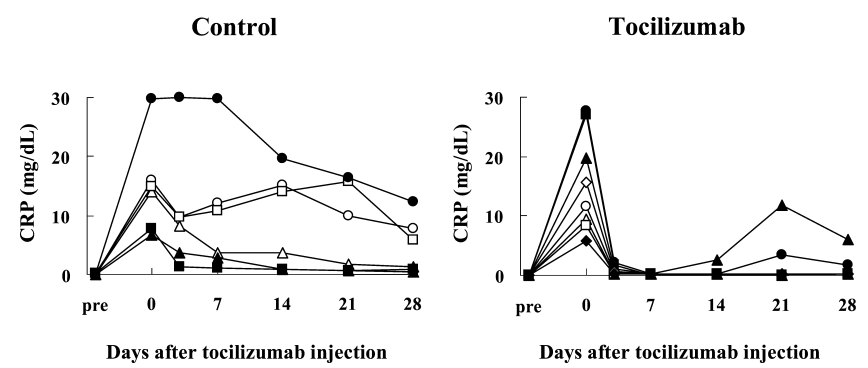

Fig. 2. CRP Levels in Tocilizumab-Treated Monkeys

Bleeding was carried out 6 times during dosing (Days 0, 3, 7, 14, 21, 28). Blood was drawn from the femoral vein with a syringe containing heparin sodium. CRP was measured by means of an automatic analyzer. Each symbol indicates the same individual shown in Fig. 1. tocilizumab antibody was detected in 7 animals (5 animals from Day 14, 1 animal from Day 21, and 1 animal on Day $21)$. Especially in 2 animals, high concentrations of antitocilizumab antibody (more than $100 \mu \mathrm{g} / \mathrm{ml}$ ) were noted from Day 21 (Table 2).

Histopathological Study The degree of neutrophil infiltration into 8 PIP joints of left hand and foot were examined histologically at the end of experiment. In the tocilizumab-
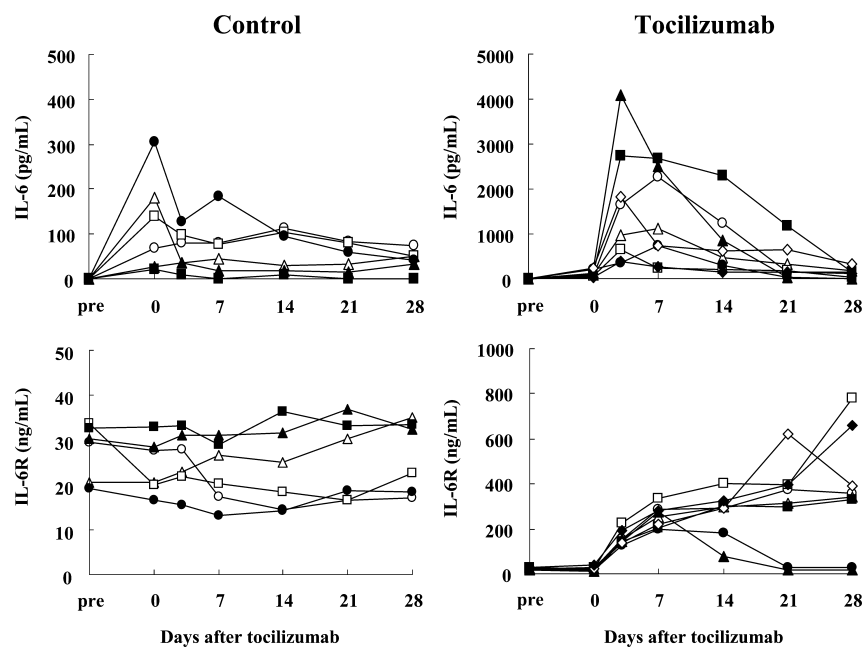

Fig. 3. Blood IL-6 and IL-6R Levels in Tocilizumab-Treated Monkeys

CIA was induced by 3 -week interval immunization of bovine type II collagen emulsified in Freund's complete adjuvant. After the onset of arthritis, tocilizumab $(30 \mathrm{mg} / \mathrm{kg})$ was injected weekly for 4 weeks. The control group received PBS in the same manner. Bleeding was carried out 6 times during dosing (Days $0,3,7,14,21,28$ ). Blood was drawn from the femoral vein with a syringe containing heparin sodium. Concentration of IL-6 and IL-6R were measured by ELISA. Each symbol indicates the same individual shown in Fig. 1.

Table 1. Effect on Biochemical Parameters

\begin{tabular}{|c|c|c|c|c|c|c|c|}
\hline & & Day 0 & Day 3 & Day 7 & Day 14 & Day 21 & Day 28 \\
\hline \multirow[t]{2}{*}{ AST (IU/1) } & Control & $32 \pm 5$ & $25 \pm 6$ & $23 \pm 4$ & $25 \pm 5$ & $23 \pm 2$ & $22 \pm 3$ \\
\hline & Tocilizumab & $32 \pm 10$ & $29 \pm 6$ & $32 \pm 7$ & $33 \pm 7$ & $30 \pm 6$ & $27 \pm 6$ \\
\hline \multirow[t]{2}{*}{$\operatorname{ALT}(\mathrm{IU} / 1)$} & Control & $19 \pm 4$ & $17 \pm 4$ & $16 \pm 4$ & $20 \pm 9$ & $18 \pm 6$ & $20 \pm 7$ \\
\hline & Tocilizumab & $22 \pm 8$ & $26 \pm 10$ & $28 \pm 16$ & $30 \pm 16$ & $32 \pm 16$ & $27 \pm 10$ \\
\hline \multirow[t]{2}{*}{ T-cho (mg/dl) } & Control & $87 \pm 28$ & $76 \pm 21$ & $76 \pm 18$ & $78 \pm 20$ & $78 \pm 23$ & $79 \pm 23$ \\
\hline & Tocilizumab & $91 \pm 21$ & $83 \pm 15$ & $78 \pm 17$ & $84 \pm 15$ & $92 \pm 20$ & $93 \pm 17$ \\
\hline \multirow[t]{2}{*}{ LDH (IU/1) } & Control & $760 \pm 95$ & $687 \pm 160$ & $567 \pm 100$ & $602 \pm 80$ & $585 \pm 73$ & $528 \pm 87$ \\
\hline & Tocilizumab & $743 \pm 186$ & $667 \pm 105$ & $702 \pm 116$ & $681 \pm 128$ & $628 \pm 80$ & $565 \pm 115$ \\
\hline \multirow[t]{2}{*}{ TG (mg/dl) } & Control & $80 \pm 68$ & $54 \pm 28$ & $49 \pm 31$ & $47 \pm 26$ & $38 \pm 25$ & $26 \pm 7$ \\
\hline & Tocilizumab & $53 \pm 27$ & $50 \pm 31$ & $37 \pm 19$ & $37 \pm 22$ & $30 \pm 8$ & $29 \pm 10$ \\
\hline
\end{tabular}

The each value indicates the mean \pm S.D. Control group and tocilizumab group consisted of 6 and 8 animals.

Table 2. Anti-tocilizumab Antibody Levels

Anti-tocilizumab antibody $(\mu \mathrm{g} / \mathrm{ml})$

\begin{tabular}{|c|c|c|c|c|c|c|}
\hline \multirow{2}{*}{ Animal No. } & \multicolumn{6}{|c|}{ Anti-tocilizumab antibody $(\mu \mathrm{g} / \mathrm{ml})$} \\
\hline & Day 0 & Day 3 & Day 7 & Day 14 & Day 21 & Day 28 \\
\hline 7 & - & - & - & - & - & - \\
\hline 8 & - & - & - & - & 0.45 & 0.65 \\
\hline 9 & - & - & - & - & 0.43 & - \\
\hline 10 & - & - & - & 16.00 & 109.00 & 336.00 \\
\hline 11 & - & - & - & 16.60 & 223.00 & 201.00 \\
\hline 12 & - & - & - & 0.41 & 5.36 & 6.21 \\
\hline 13 & - & - & - & 1.37 & 3.56 & 3.70 \\
\hline 14 & - & - & - & 4.48 & 2.07 & 0.65 \\
\hline
\end{tabular}

Serum levels of anti-tocilizumab antibody were measured by specific ELISA. 


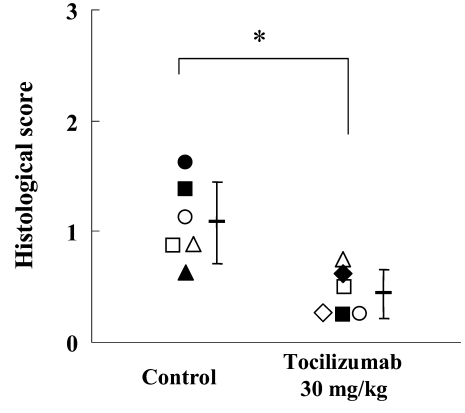

Fig. 4. Histopathology of PIP Joints

At the end of experiment, histological study was performed. Histological grading of neutrophil infiltration was carried out using a grading system of $0-3(0$ : no neutrophil infiltration, 1: very slight, 2: slight, 3 : moderate). For the analysis, two animals with high titer of anti-tocilizumab antibody were excluded. Each symbol indicates the same individual shown in Fig. 1. Statistical significance was analyzed by $U$-test $(* p<0.05)$.

treated group, there were less neutrophils in PIP joints compared with the control group (Fig. 4).

\section{DISCUSSION}

The blockade of IL- 6 by antibody or gene disruption restrained the development of arthritis in several arthritis models. $^{5-11)}$ However, in antibody-treated studies IL-6 was blocked at the time of arthritis induction, and in the IL-6-deficient mouse studies IL-6 was absent from the development. Namely, these studies never examined the direct influence of IL-6 blockade on joint swelling. Regarding therapeutic effect of IL- 6 blockade, we examined the efficacy of anti-mouse IL-6R antibody after the onset of arthritis in several mouse arthritis models such as CIA and anti-collagen antibody-induced arthritis. But anti-IL-6R antibody did not affect joint swelling (unpublished data). In the present study, we finally found that IL-6 inhibition ameliorated joint swelling when tocilizumab was injected after the onset of arthritis in monkey CIA.

Rodent CIA model is widely used for the evaluation of anti-rheumatic drugs and the analysis of RA pathogenesis. The result that tocilizumab reduced joint swelling was very surprising because anti-IL-6R antibody did not work after the onset of arthritis in rodent CIA. The reason of the discrepant result between monkey CIA and mouse CIA is unclear. Joint swellings in monkey CIA last more than one month. However, those in rodent CIA are transient. From these facts, IL-6 may be involved in the maintenance of arthritis in monkey CIA. Anyway monkey CIA could help to bridge the gap between rodent models and human patients regarding the involvement of IL-6.

Tocilizumab treatment reduced the number of neutrophil in the joints. By HE stain, neutrophil is easily identified morphologically, but other inflammatory cells such as T cells, B cells and monocytes were not. In the preliminary observation, however, the total numbers of cells in the joint were also reduced by tocilizumab. Inflammatory cells infiltrate into joints and play an essential role in the development and maintenance of inflammation. Therefore, it is thought that the inhibition of inflammatory cell infiltration into joint involves in the reduction of swelling of joint.

Anti-inflammatory mechanism of tocilizumab on established arthritis is not fully understood. IL-6 induces mono- cyte chemotactic protein-1 (MCP-1) from monocytes and the IL-6/IL-6R complex induces MCP-1 and adhesion molecules, ICAM-1 in endothelial cells. ${ }^{12,13)}$ These lines of evidence support the idea that the IL-6 system plays a positive role in the local inflammatory reaction by amplifying inflammatory cell infiltration. Furthermore, angiogenesis is a characteristic feature of RA synovium. Newly formulated blood vessels are used for the infiltration of inflammatory cells, and support the growth of synovial cells. IL-6 up-regulates the production of vascular endothelial growth factor (VEGF), which plays important roles in angiogenesis in synovium. ${ }^{14)}$ Tocilizumab may exert its anti-inflammatory effect via the inhibition of these IL- 6 biological activities.

It is reported that in the patients with RA and Crohn's disease, the administration of tocilizumab increased blood levels of IL-6 and sIL-6R. ${ }^{15,16)}$ In this monkey CIA model, blood IL-6 and sIL-6R levels rose by the injection of tocilizumab (Fig. 3). IL-6 level rapidly increased after tocilizumab injection, and thereafter it decreased. This phenomenon is explained as follows. Tocilizumab can inhibit IL- 6 binding to IL-6R and dissociate IL-6/sIL-6R complex, therefore, IL-6 is accumulated in the blood. And IL-6 production was reduced in accompanied with the improvement of arthritis. As a result, blood IL-6 level decreases. On the other hand, sIL-6R level rapidly increased after tocilizumab injection, but thereafter it remained or further increased. As tocilizumab exists as a complex with sIL-6R in the blood (unpublished data), the clearance from blood is delayed by the formation of complex. The further studies to analyze the mechanism of IL-6 and IL-6R elevation by tocilizumab are now underway.

It is reported that the blood concentration of liver enzymes such as ALT and AST, and cholesterol rose in tocilizumabtreated RA patients. ${ }^{2)}$ We examined whether a similar phenomenon was seen in monkey arthritis model. However, as shown in Table 1, the elevation of such biochemical parameters was not recognized in tocilizumab-treated animals. The reason why such a difference was provided between humans and monkeys is unknown.

In case of RA patients, monthly injection of $8 \mathrm{mg} / \mathrm{kg}$ tocilizumab significantly ameliorated disease activity and anti-tocilizumab antibody rarely induced at this administration dose. ${ }^{2-4)}$ However, in this study we injected weekly $30 \mathrm{mg} / \mathrm{kg}$ of tocilizumab, because we previously showed that higher dose of tocilizumab inhibited the induction of antitocilizumab antibody in monkeys. ${ }^{5)}$ In spite of it, 7/8 animals produced anti-tocilizumab antibody. This fact strongly suggests that IL-6 does not play a role in anti-tocilizumab antibody production, despite IL-6 is originally reported to augment antibody production. ${ }^{17)}$

We here demonstrated that tocilizumab improved established arthritis and CIA in monkey is useful for the analysis of anti-arthritic effect of tocilizumab. Now we are underway to examine the mechanism of anti-arthritic action of tocilizumab using monkey CIA.

\section{REFERENCES}

1) Mihara M., Kasutani K., Okazaki M., Nakamura A., Kawai S., Sugimoto M., Matsumoto Y., Ohsugi Y., Int. Immunopharmacol., 5, $1731-1740$ (2005).

2) Nishimoto N., Yoshizaki K., Miyasaka N., Yamamoto K., Kawai S., 
Takeuchi T., Hashimoto J., Azuma J., Kishimoto T., Arthritis Rheum., 50, 1761-1769 (2004).

3) Maini R. N., Taylor P. C., Szechinski J., Pavelka K., Bröll J., Balint G., Emery P., Raemen F., Petersen J., Smolen J., Thomson D., Kishimoto T., Arthritis Rheum., 54, 2817-2829 (2006).

4) Nishimoto N., Hashimoto J., Miyasaka N., Yamamoto K., Kawai S., Takeuchi T., Murata N., van der Heijde D., Kishimoto T., Ann. Rheum. Dis., 66, 1162-1167 (2007)

5) Mihara M., Kotoh M., Nishimoto N., Oda Y., Kumagai E., Takagi N., Tsunemi K,, Ohsugi Y,, Kishimoto T,, Yoshizaki K,, Takeda Y., Clin. Immunol., 98, 319-326 (2001).

6) Takagi N., Mihara M., Moriya Y., Nishimoto N., Yoshizaki K., Kishimoto T., Takeda Y., Ohsugi Y., Arthritis Rheum., 41, 2117-2121 (1998).

7) Sasai M., Saeki Y., Ohshima S., Nishioka K., Mima T., Tanaka T., Katada Y., Yoshizaki K., Suemura M., Kishimoto T., Arthritis Rheum., 42, 1635-1643 (1999).

8) Boe A., Baiocchi M., Carbonatto M., Papoian R., Serlupi-Crescenzi O., Cytokine, 11, 1057-1064 (1999).

9) Kagari T., Doi H., Shimozato T., J. Immunol., 169, 1459-1466 (2002).

10) Ishihara K., Sawa S., Ikushima H., Hirota S., Atsumi T., Kamimura D.,
Park S. J., Murakami M., Kitamura Y., Iwakura Y., Hirano T., Int. Immunol., 16, 455-465 (2004).

11) Hata H., Sakaguchi N., Yoshitomi H., Iwakura Y., Sekikawa K., Azuma Y., Kanai C., Moriizumi E., Nomura T., Nakamura T., Sakaguchi S., J. Clin. Invest., 114, 582-588 (2004).

12) Biswas P., Delfanti F., Bernasconi S., Mengozzi M., Cota M., Polentarutti N., Mantovani A., Lazzarin A., Sozzani S., Poli G., Blood, 91 , $258-265$ (1998).

13) Romano M., Sironi M., Toniatti C., Polentarutti N., Fruscella P., Ghezzi P., Faggioni R., Luini W., van Hinsbergh V., Sozzani S., Bussolino F., Poli V., Ciliberto G., Mantovani A., Immunity, 6, 315-325 (1997).

14) Nakahara H., Song J., Sugimoto M., Hagihara K., Kishimoto T., Yoshizaki K., Nishimoto N., Arthritis Rheum., 48, 1521-1529 (2003).

15) Ito H., Takazoe M., Fukuda Y., Hibi T., Kusugami K., Andoh A., Matsumoto T., Yamamura T., Azuma J., Nishimoto N., Yoshizaki K., Shimoyama T., Kishimoto T., Gastroenterology, 126, 989—996 (2004).

16) Nishimoto N., Terao K., Kakehi T., Kishimoto T., Arthritis Rheum., 50, S109 (2004).

17) Muraguchi A., Hirano T., Tang B., Matsuda T., Horii Y., Nakajima K., Kishimoto T., J. Exp. Med., 167, 332-344 (1988). 